\title{
Is it worth closing the atrial septal defect in patients with insignificant shunt?
}

\author{
Monika Komar, Tadeusz Przewłocki, Maria Olszowska, Bartosz Sobień, Lidia Tomkiewicz-Pająk, Piotr Podolec \\ Department of Cardiac and Vascular Diseases, Institute of Cardiology, Jagiellonian University, Krakow, Poland
}

Postep Kardiol Inter 2014; 10, 2 (36): 78-83

DOI: $10.5114 /$ pwki.2014.43510

\begin{abstract}
A bstract
Introduction: Closure of the atrial septal defect in patients with insignificant shunt is controversial.

Aim: To evaluate the outcomes of transcatheter closure of atrial septal defect (ASD) in symptomatic patients with borderline shunt.

Material and methods: One hundred and sixty patients (120 female, 40 male) with a mean age of $30.1 \pm 16.2$ (20-52) years with a small ASD who underwent transcatheter closure were analyzed. All patients had a small ASD with Qp : Qs ratio $\leq 1.5$, mean $1.2 \pm 0.9(1.1-1.5)$ in echo examination. Cardiopulmonary exercise tests, clinical study, transthoracic echocardiographic study as well as quality of life (QoL) (measured using the SF36 questionnaire (SF36q)) were repeated in all patients before and after the procedure.

Results: The devices were successfully implanted in all patients. After 12 months of ASD closure, all the patients showed a significant improvement of exercise capacity (oxygen consumption $-21.9 \pm 3.1 \mathrm{vs.} 30.4 \pm 7.7, p>0.001$ ). The QoL improved in 7 parameters at 12-month follow-up. The mean SF36q scale increased significantly in 141 (88.1\%) patients of mean $43.2 \pm 20.1$ (7-69). A significant decrease of the right ventricular area $\left(20.3 \pm 1.3 \mathrm{~cm}^{2} \mathrm{vs} .18 .3 \pm 1.2 \mathrm{~cm}^{2}, p<0.001\right)$ and the right atrial area $\left(15.2 \pm 1.9 \mathrm{~cm}^{2} \mathrm{vs} .12 .0 \pm 1.6 \mathrm{~cm}^{2}\right.$, $p<0.001)$ was observed at 12-month follow-up.

Conclusions: Closure of ASD in the patients with insignificant shunt resulted in significant durable clinical and hemodynamic improvement after percutaneous treatment.
\end{abstract}

Key words: atrial septal defect, transcatheter closure, cardiopulmonary exercise test, quality of life.

\section{Introduction}

Atrial septal defect (ASD) not uncommonly remains undiagnosed until adulthood. The most common presenting symptoms at adult age are palpitations and exercise intolerance manifested as either exertional dyspnea or fatigue, which increases with age [1-3]. More serious complications are typically seen in older patients with previously unrecognized ASD who have been exposed to large left-to-right shunting for a long period [4, 5].

Patients with a hemodynamically significant ASD could be offered surgical or percutaneous closure of the ASD.

Recently, the definition of "significant ASD" has been changed. Nowadays significant ASD, according to the ESC Guidelines, is defined as shunt with signs of right ventricular volume overload despite the Qp : Qs ratio [1, 4, 5]. Patients with significant shunt (signs of right ventricle (RV) volume overload) and pulmonary vascular resistance
$<5$ Wood units should undergo ASD closure regardless of symptoms [5].

Still the closure of the ASD in patients with insignificant shunt (with Qp : Qs ratio less than $1.5: 1$ ) and lack of pulmonary overload and hypertension is controversial.

Aim

The aim of the present study was to evaluate the outcomes of transcatheter closure of secundum ASD in patients with a Qp : Qs ratio less than 1.5 : 1 .

\section{Material and methods}

\section{Patient population}

Out of a total of 488 consecutive patients with ASD who underwent transcatheter closure over 10 years in our department 160 patients (120 female, 40 male), mean age of $30.1 \pm 16.2$ (20-52) years, with insignificant

\section{Corresponding author:}

Monika Komar MD, PhD, Department of Cardiac and Vascular Diseases, Institute of Cardiology, Medical College, Jagiellonian University, 80 Prądnicka St, 31-202 Krakow, Poland, phone: +48 1261422 87, fax: +48 1242343 76, e-mail: moni_s@interia.pl

Received: 10.04.2014, accepted: 8.05.2014. 
shunt were analyzed. The patients had an isolated secundum ASD with a left-to-right shunt (ratio of pulmonary to systemic blood flow or Qp : Qs $<1.5$ : 1 mean $1.2 \pm 0.9$ (1.1-1.5) in echo examination). All those patients had coexisting impairment of physical status or enlargement of the right heart cavity or pulmonary hypertension.

The major demographic characteristics of patients are summarized in Table I.

\section{Protocol}

A detailed description of the transcatheter closure technique has been given previously $[4,5]$. The procedure was performed under local anesthesia. Before the procedure, a hemodynamic study was performed for all the patients. Left-to-right shunt as well as pulmonary artery pressure was evaluated by echocardiography and verified during cardiac catheterization. All procedures were performed with continuous transesophageal echocardiographic monitoring.

After procedure the patients were treated with $75 \mathrm{mg}$ acetylsalicylic acid and $75 \mathrm{mg}$ clopidogrel once daily for 1 month and then $325 \mathrm{mg}$ acetylsalicylic acid for 11 months to provide antithrombotic protection until full device endothelialization.

The assessment was made in compliance with the following constraints:

- clinical study - within 7 days before the procedure and then at 1, 6 and 12 months after,

- transthoracic echocardiographic examination - within 7 days before the procedure and then at 1, 6 and 12 months after,

- cardiopulmonary exercise test - within 7 days before the procedure and then at 6 and 12 months after,

- quality of life measurement - within 7 days before the procedure and then at 12 months after.

\section{Clinical study}

The clinical study was based on subjective opinion of the patients concerning their functional capacity. We assessed the frequency of dyspnea incidents and palpitations.

\section{Transthoracic echocardiographic examination}

The transthoracic echocardiographic examination (TTE) study was performed using a Toshiba Power Vision machine with a 5.0-MHz multiplane probe, according to a standard protocol including color flow Doppler data. After a comprehensive echocardiographic study, right ventricular, right atrial major and minor dimensions, and left ventricular dimensions were measured in end-diastole. M-mode echocardiography from the parasternal short-axis view was used to measure right ventricular dimensions. The apical four-chamber view was used to measure the right atrial and ventricle size. The major axis of the right atrium was measured from the tricuspid annulus to the
Table I. Demographic characteristics of patients

$$
(n=160)
$$

\begin{tabular}{lc} 
Parameter & $\begin{array}{c}\text { Results } \\
n(\%)\end{array}$ \\
\hline Smoking & $20(12.5)$ \\
\hline Obesity & $16(10)$ \\
\hline Diabetes mellitus & $4(2.5)$ \\
\hline Hypertension & $45(28.1)$ \\
\hline Dyslipidemia & $51(31.9)$ \\
\hline Coronary artery disease & $1(0.6)$ \\
\hline Atrial fibrillation & $17(10.6)$ \\
\hline Pulmonary hypertension* & $16(10)$
\end{tabular}

Measured in right-heart catheterization: pulmonary hypertension - mean pulmonary artery pressure $\geq 25 \mathrm{~mm} \mathrm{Hg}$

atrial superior wall and the minor axis was measured perpendicular to the major axis at half of the length of the latter. Each measurement was averaged from 5 consecutive cardiac cycles. Two independent cardiologists trained in echocardiography agreed on the measurements. Follow-up included a complete echocardiographic study with a precise location and measurement of any residual shunt as well as position of the device.

\section{Cardiopulmonary exercise test}

In order to objectively assess the clinical and functional status of the patients in all the cases we performed cardiopulmonary exercise tests.

Maximal cardiopulmonary testing was performed on an ergometer cycle. Peak oxygen uptake, carbon dioxide production, and minute ventilation were measured with a computerized breath-by-breath analyzer (V-MAX 29, Sensor-Medics). Patients performed a maximal exercise test using a 1-min incremental bicycle protocol with a work rate increment of $10 \mathrm{~W} / \mathrm{min}$. Criteria for test ending were considered patient exhaustion or a respiratory exchange ratio $\geq 1.09$. A 12 -lead electrocardiogram was also monitored throughout the study, and cuff blood pressure was determined manually every 2 min. Standard equations were used to generate predicted values for baseline spirometric and peak exercise parameters.

\section{Quality of life}

This objective study was completed with the subjective opinions of the patients concerning their clinical status. In each case we assessed the quality of life using the SF36 questionnaire. The patients completed the questionnaire SF36 - the best-known questionnaire in measuring health status. The number " 36 " refers to 36 questions which concern 8 aspects of general health and quality of life - that is: physical function, role-physical, bodily pain, general health, vitality, social function, role-emotional and mental health. 


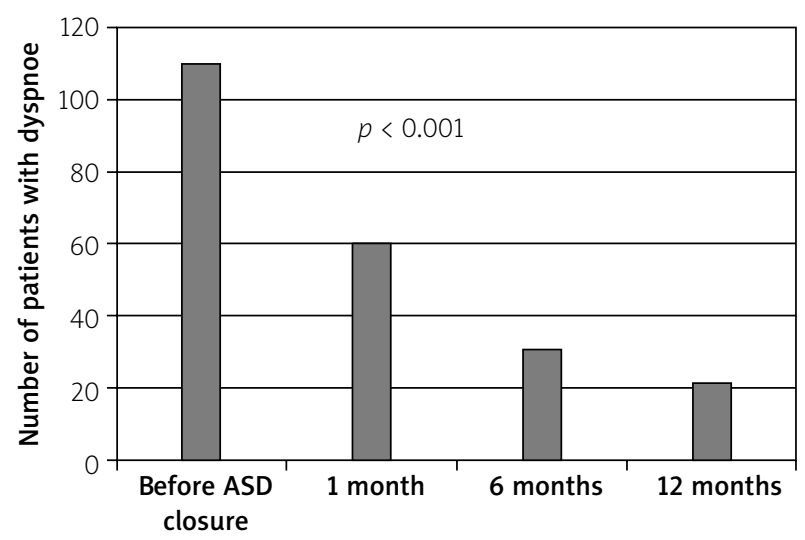

Figure 1. Frequency of dyspnea before and after ASD closure

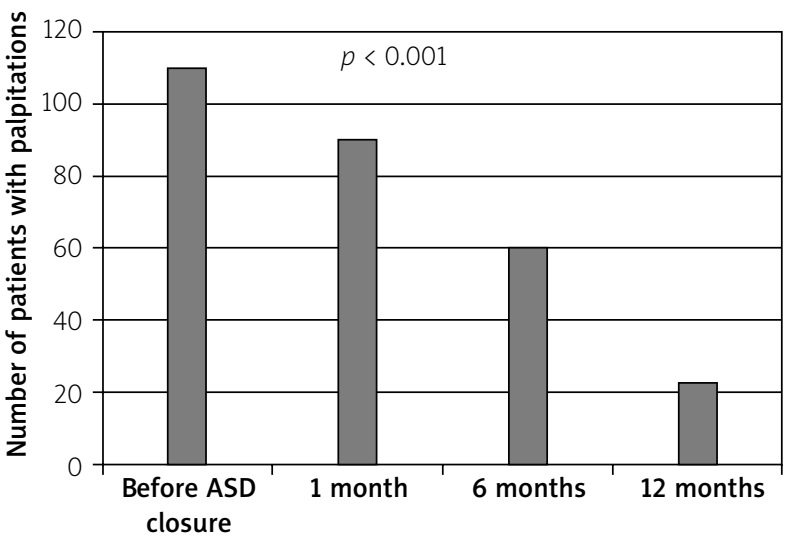

Figure 2. Frequency of palpitations before and after ASD closure

\section{Statistical analysis}

Continuous data are expressed as mean \pm standard deviation and baseline and follow-up were compared by the paired Student's $t$-test. For the comparison of categorical variables the $\chi^{2}$ test was used. The level of statistical significance was two-sided and set at $p<0.05$. Statistical analysis was performed with SPSS version 15.0.0 for Windows.

\section{Results}

The left-to-right shunt (Qp : Qs) in hemodynamic evaluation before ASD closure was comparable to measurement of Qp : Qs in the echo examination (1.3 \pm 0.6 (1.1-1.6) vs. $1.2 \pm 0.9$ (1.1-1.5), $p=0.598)$. Pulmonary hypertension measured in right-heart catheterization was found in 16 (10\%) patients (mean PAP $16 \pm 3.5$ (11-24), systolic PAP $29 \pm 7.5$ (19-35)).
The defect echo diameter was $7.0 \pm 3.4$ (4-15) $\mathrm{mm}$. The mean balloon stretched diameter of ASD was 9.6 $\pm 6.1 \mathrm{~mm}(6-18 \mathrm{~mm})$.

The devices were successfully implanted in all the subjects (procedure time $18.8 \pm 4.8$ (9-35) min, fluoroscopy time $8.5 \pm 4.9(6-13) \mathrm{min})$. The diameter of the implanted devices ranged from $8 \mathrm{~mm}$ to $20 \mathrm{~mm}$. The Amplatzer ASD closure device was used in 105 (65.6\%) patients, the Starflex device in 10 (6.3\%) patients, and Cardia ASD device in 45 (28.1\%) patients.

Minor complications were recorded in 15 (9.4\%) patients with minor venous access bleeding without transfusion. In 25 (15.6\%) cases transient rhythm disorders were noted. There were no serious complications involved, no fractures of the device, and no device embolizations.

All the patients remained well with no complications or new symptoms at their 12-month follow-up. No patient has had any heart-related hospital admissions. All the patients had complete 12-months follow-up.

\section{Clinical study}

Transcatheter closure of secundum ASD has created significant improvement of the clinical status of the patients in the early follow-up. It caused reduction of the breathlessness and palpitation as early as a month after the procedure (Figures 1 and 2).

\section{Transthoracic echocardiographic examination}

Directly after the procedure transthoracic echocardiography showed residual trivial shunt in 13 (9.3\%) subjects, which disappeared in all cases within the 6-month follow-up period.

As early as 1 month after the procedure, a significant decrease of the right ventricular dimension and the right atrial dimension was observed. All the analyzed dimensions of the right heart significantly decreased (Table II).

\section{Cardiopulmonary exercise tests}

Significant improvement of exercise capacity was noted at 6 and 12 months after the procedure. The time of exercise within 6 months of ASD closure was longer, as compared to the baseline values and also the oxygen consumption increased (Table III).

\section{Quality of life}

Apart from the objective improvement of physical activity in the cardiopulmonary exercise test, significant improvement of the quality of life in patients based on the SF36 form was also noted. Moreover, an improvement of each aspect of quality of life with the exception of mental health was observed. The mean SF36q scale increased significantly in $141(88.1 \%)$ patients of mean $43.2 \pm 20.1$ (7-69). The total SF36 scale improved from 34.2 to 70.2 points (Table IV). 
Table II. Echocardiographic parameters before and after ASD closure

\begin{tabular}{lccccccc}
\multicolumn{1}{|c}{ Parameter } & $\begin{array}{c}\text { Before ASD } \\
\text { closure }\end{array}$ & $\begin{array}{c}1 \text { month after } \\
\text { ASD closure }\end{array}$ & $\begin{array}{c}6 \text { months } \\
\text { after ASD } \\
\text { closure }\end{array}$ & $\begin{array}{c}12 \text { months } \\
\text { after ASD } \\
\text { closure }\end{array}$ & $\begin{array}{c}\text { Value of } \\
p \text { before } \\
\text { vs. } 1 \text { month }\end{array}$ & $\begin{array}{c}\text { Value of } \\
p \\
\text { before } \\
\text { vs. } 6 \text { months }\end{array}$ & $\begin{array}{c}\text { Value of } \\
p \\
\text { before } \\
\text { vs. 12 } \\
\text { months after }\end{array}$ \\
\hline $\mathrm{RV}_{\text {diastole }}[\mathrm{mm}]$ & 31.4 & 26.9 & 26.6 & 25.2 & $<0.001$ & $<0.001$ & $<0.001$ \\
\hline $\mathrm{RV}_{\text {systole }}[\mathrm{mm}]$ & 26.9 & 22.1 & 21.2 & 21.1 & $<0.001$ & $<0.001$ & $<0.001$ \\
\hline $\mathrm{RV}_{\text {long axis }}[\mathrm{mm}]$ & 67.2 & 64.9 & 64.9 & 65.0 & $<0.001$ & $<0.001$ & $<0.001$ \\
\hline $\mathrm{RV}_{\text {short axis }}[\mathrm{mm}]$ & 41.6 & 33.9 & 31 & 30.8 & $<0.001$ & $<0.001$ & $<0.001$ \\
\hline $\mathrm{RV}_{\text {area }}\left[\mathrm{cm}^{2}\right]$ & 20.3 & 21 & 19.9 & 18.3 & $<0.001$ & $<0.001$ & $<0.001$ \\
\hline $\mathrm{RA}_{\text {ong axis }}[\mathrm{mm}]$ & 48.1 & 42.2 & 39.0 & 39.1 & $<0.001$ & $<0.001$ & $<0.001$ \\
\hline $\mathrm{RA}_{\text {short axis }}[\mathrm{mm}]$ & 40.1 & 34.1 & 33.0 & 33.0 & $<0.001$ & $<0.001$ & $<0.001$ \\
\hline $\mathrm{RA}_{\text {area }}\left[\mathrm{cm}^{2}\right]$ & 15.2 & 14 & 12.9 & 12.0 & $<0.001$ & $<0.001$ & $<0.001$
\end{tabular}

Table III. Parameters of the cardiopulmonary exercise test before and after ASD closure

\begin{tabular}{|c|c|c|c|c|}
\hline Parameter & Before ASD closure & $\begin{array}{c}6 \text { months after ASD } \\
\text { closure }\end{array}$ & $\begin{array}{c}12 \text { months after ASD } \\
\text { closure }\end{array}$ & $\begin{array}{l}\text { Value of } p \text { before vs. } \\
12 \text { months after }\end{array}$ \\
\hline Time of exercise $[\mathrm{s}]$ & $689 \pm 108$ & $789 \pm 113$ & $903 \pm 175$ & $<0.001$ \\
\hline Heart rate [beats/min] & $155 \pm 30$ & $164 \pm 29.8$ & $172 \pm 29$ & $<0.01$ \\
\hline Heart rate $\%$ & $81 \pm 10.9$ & $91 \pm 13$ & $91.1 \pm 12.9$ & $<0.001$ \\
\hline $\mathrm{VO}_{2 \text { peak }}[\mathrm{ml} / \mathrm{kg} / \mathrm{min}]$ & $21.9 \pm 3.1$ & $30.1 \pm 8$ & $30.4 \pm 7.7$ & $<0.001$ \\
\hline $\mathrm{VO}_{2 \text { peak }} \%$ & $71 \pm 12.2$ & $83 \pm 17$ & $86.3 \pm 16$ & $<0.001$ \\
\hline $\mathrm{VCO}_{2}[\mathrm{l} / \mathrm{min}]$ & $1.55 \pm 0.9$ & $1.56 \pm 1.1$ & $1.83 \pm 0.8$ & $<0.001$ \\
\hline Pulse $\mathrm{O}_{2}$ & $11.1 \pm 2.0$ & $13.2 \pm 2.2$ & $13.5 \pm 2.1$ & $<0.001$ \\
\hline VD/VT & $0.22 \pm 0.1$ & $0.16 \pm 0.05$ & $0.16 \pm 0.06$ & $<0.01$ \\
\hline $\mathrm{VE} / \mathrm{VCO}_{2}$ & $31.6 \pm 3.2$ & $27 \pm 4.6$ & $26.5 \pm 4.2$ & $<0.05$ \\
\hline $\mathrm{PETO}_{2}[\mathrm{kPa}]$ & $14.1 \pm 0.9$ & $14 \pm 0.2$ & $14.2 \pm 0.8$ & NS \\
\hline $\mathrm{PETCO}_{2}[\mathrm{kPa}]$ & $4.9 \pm 0.6$ & $5.0 \pm 0.4$ & $5.0 \pm 0.1$ & NS \\
\hline $\begin{array}{l}\mathrm{T}_{\mathrm{A}}[\mathrm{s}] \text { - time to attain anaerobic } \\
\text { treasure }\end{array}$ & $413 \pm 101$ & $524 \pm 139$ & $532 \pm 125$ & $<0.001$ \\
\hline $\mathrm{VO}_{2 \mathrm{AT}}[\mathrm{ml} / \mathrm{kg} / \mathrm{min}]$ & $16.0 \pm 12.02$ & $17.1 \pm 14.8$ & $19.8 \pm 14.7$ & $<0.001$ \\
\hline $\mathrm{VO}_{2 \mathrm{AT}} \%$ & $44 \pm 11.9$ & $45.2 \pm 11.0$ & $50 \pm 11.6$ & $<0.001$ \\
\hline
\end{tabular}

$\mathrm{VO}_{2 \text { peak }}$ - peak oxygen consumption, $\mathrm{VCO}_{2}$ - production of carbon dioxide, pulse $\mathrm{O}_{2}$-oxygen pulse, VD/VT - tidal volume, VENCOO - ventilatory equivalent for CO TAT - time to attain anaerobic treasure, $\mathrm{VO}_{2 A T}$ - oxygen consumption at anaerobic threshold

The canonical correlation analysis showed that the following parameters had an influence on improvement of the total SF36 scale: improvement in cardiopulmonary test (prolonged time of exercise, improvement of $\mathrm{VO}_{2 \text { peak }}$ ( $\mathrm{ml} / \mathrm{kg} / \mathrm{min})), p<0.05$. The right-to-left shunt (Qp : Qs) before ASD closure had no influence on improvement of the total SF36 scale $(p<0.621)$.

The multiple forward stepwise regression analysis revealed that of all parameters (parameters of echocardiog- raphy study and cardiopulmonary exercise test) reduction in right heart size after ASD closure $\left(\delta R V_{\text {area }}\left(\mathrm{cm}^{2}\right) ; F(6.31)=\right.$ 9.0141; $p<0.001$, standard error 2.210) had the strongest influence on improvement of the total SF36 scale.

\section{Discussion}

Isolated ASD constitutes the second most common (after bicuspid aortic valve) congenital heart lesion in adults [1, 2]. 
Table IV. Results of the SF 36 questionnaire before and after ASD closure

\begin{tabular}{lccc} 
Parameter & Before ASD closure & 12 months after & Value of $p$ \\
\hline SF36 Total scale & $34.2 \pm 4.9$ & $70.2 \pm 8.9$ & $<0.001$ \\
\hline PF - Physical function & $8.2 \pm 1.1$ & $10.2 \pm 4.2$ & $<0.01$ \\
\hline RP - Role physical & $6.1 \pm 2.7$ & $10.1 \pm 1.1$ & $<0.01$ \\
\hline BP - Bodily pain & $7.0 \pm 4.0$ & $14.2 \pm 4.0$ & $<0.01$ \\
\hline GH - General health & $4.2 \pm 4.9$ & $11.9 \pm 4.0$ & $<0.001$ \\
\hline V - Vitality & $7.2 \pm 3.3$ & $11.2 \pm 3.3$ & $<0.01$ \\
\hline SF - Social function & $9.2 \pm 3.9$ & $14.2 \pm 4.5$ & $<0.01$ \\
\hline RE - Role emotional & $14.1 \pm 7.3$ & $19.1 \pm 8.9$ & $<0.01$ \\
\hline MH - Mental health & $4.2 \pm 4.9$ & $4.7 \pm 4.0$ & 0.78
\end{tabular}

Surgical closure of ASD has been practiced for about 45 years [3, 4]. Since the first attempt in 1976, transcatheter closure of secundum ASD has evolved over the past three decades [5-8]. and now according to guidelines is the therapy of choice when possible [8].

The benefits of transcatheter treatment of congenital heart diseases are obvious. First of all, we can avoid complications of open heart surgery, which is absolutely crucial in the group of patient with insignificant shunt. Another benefit is avoidance of a surgical scar commonly called by psychologists a "scar of the human soul" and shorter hospitalization time [8, 9]. It allows even percutaneously, the closure of the ASD in the patients with insignificant shunt is controversial. Some authors undermine the necessity to correct atrial communications in those cases, giving the argument that the risk involved in the procedure is greater than its benefit.

However, based on some analyses, it would appear that ASD should be closed when they are identified, irrespective of the patient's symptoms [10-14].

"Significant ASD" (defined as shunt with pulmonary to systemic blood flow Qp : Qs over 1.5 : 1) has been an indication for intervention in ASD for the last several years. Nowadays significant ASD, according to the ESC Guidelines, is defined as shunt with signs of right ventricular volume overload despite Qp : Qs $[8,15]$. Also patients who are asymptomatic or mildly symptomatic should be offered defect closure, because the natural course of untreated ASD often leads to a shortened life expectancy compared with healthy subjects $[16,17]$. Brochu et al. showed that even adult ASD patients classified as asymptomatic showed a significant increase in their functional capacity after percutaneous closure [18].

The aim of our study was to evaluate the results of transcatheter closure of ASD in patients with insignificant shunt defined as shunt with Qp : Qs $<1.5$ : 1. All those patients had coexisting subjective impairment of physical status or enlargement of the right heart cavity or pulmonary hypertension.

In our conviction patients with a small ASD without clinical symptoms and with normal right heart and nor- mal pulmonary artery pressure may remain under medical observation as there is no reason to close the ASD. Conversely, patients with clinical impairment and/or right heart enlargement could benefit from ASD closure.

In our patients we found a significant reduction in right heart dimension after device closure. Nearly all patients showed reduction of symptoms and an improvement in functional and exertional capacity in cardiopulmonary exercise tests. The quality of life measured by the SF36 test improved. Improvement in exercise capacity had the greatest influence on the improvement of quality of life in our patients. Moreover, improvement in exercise capacity was correlated with better quality of life after ASD closure. On the other hand, the left-to-right shunt before ASD closure had no influence on improvement of the quality of life. Even the patients with the minimal shunt benefit from ASD closure, in terms of quality of life and exercise capacity.

Our analysis revealed that of all analyzed parameters only reduction in right heart size after ASD closure had a significant influence on improvement of the total SF36 scale as well as exercise capacity. This therefore suggests that in patients with insignificant shunt, enlargement of the right ventricle should be one of the main criteria for ASD closure.

Our study results confirm that device closure of ASD is safe with minimal complications, which is consistent with the reports in the literature [18-22].

Thus we are convinced that it is really worth closing ASD despite the Qp : Qs ratio in patients with coexisting impairment of physical status or enlargement of the right heart cavity.

Of course, each case should be analyzed and qualified individually, and other reasons for physical impairment should be excluded, especially in older patients.

\section{Conclusions}

Transcatheter closure of ASD in symptomatic patients with borderline shunt is associated with marked clinical, functional and quality of life improvement. 


\section{References}

1. Campbell M. Natural history of atrial septal defect. Br Heart J 1970; 32: 820-6.

2. Hoffman JI, Kaplan S. The incidence of congenital heart disease. J Am Coll Cardiol 2002; 39: 1890-900.

3. Murphy JG, Gersh BJ, McGoon MD, et al. Long-term outcome after surgical repair of isolated atrial septal defect. Follow-up at 27 to 32 years. N Engl J Med 1990; 323: 1645-50.

4. Butera G, Carminati M, Chessa M, et al. Percutaneous versus surgical closure of secundum atrial septal defect: comparison of early results and complications. Am Heart J 2006; 151: 228-34.

5. Fischer G, Stieh J, Uebing A, et al. Experience with transcatheter closure of secundum atrial septal defects using the Amplatzer septal occluder: a single centre study in 236 consecutive patients. Heart 2003; 89: 199-204.

6. Amin Z, Hijazi ZM, Bass JL, et al. Erosion of Amplatzer septal occluder device after closure of secundum atrial septal defects: review of registry of complications and recommendations to minimize future risk. Catheter Cardiovasc Interv 2004; 63: 496-502.

7. Masura J, Gavora P, Podnar T. Long-term outcome of transcatheter secundum-type atrial septal defect closure using Amplatzer septal occluders. J Am Coll Cardiol 2005; 45: 505-7.

8. Veldtman GR, Razack V, Siu S, et al. Right ventricular form and function after percutaneous atrial septal defect device closure. J Am Coll Cardiol 2001; 37: 2108-13.

9. Attie F, Rosas M, Granados N, et al. Surgical treatment for secundum atrial septal defects in patients $>40$ years old. A randomized clinical trial. J Am Coll Cardiol 2001; 38: 2035-42.

10. Steele PM, Fuster V, Cohen M, et al. Isolated atrial septal defect with pulmonary vascular obstructive disease - long-term follow-up and prediction of outcome after surgical correction. Circulation 1987; 76: 1037-42.

11. Berger F, Vogel M, Kramer A, et al. Incidence of atrial flutter/ fibrillation in adults with atrial septal defect before and after surgery. Ann Thorac Surg 1999; 68: 75-8.

12. Alfakih K, Plein S, Thiele $\mathrm{H}$, et al. Normal human left and right ventricular dimensions for MRI as assessed by turbo gradient echo and steady-state free precession imaging sequences. J Magn Reson Imaging 2003; 17: 323-9.

13. Hauser TH, McClennen S, Katsimaglis G, et al. Assessment of left atrial volume by contrast enhanced magnetic resonance angiography. J Cardiovasc Magn Reson 2004; 6: 491-7.

14. Harper RW, Mottram PM, McGaw DJ. Closure of secundum atrial septal defects with the Amplatzer septal occluder device: techniques and problems. Catheter Cardiovasc Interv 2002; 57: 508-24.

15. Baumgartner H, Bonhoeffer P, De Groot NMS, et al. ESC Guidelines for the management of grown-up congenital heart disease (new version 2010). Eur Heart J 2010; 31: 2915-57.

16. Grothues F, Moon JC, Bellenger NG, et al. Interstudy reproducibility of right ventricular volumes, function, and mass with cardiovascular magnetic resonance. Am Heart J 2004; 147: 218-23.

17. Galal MO, Wobst A, Halees Z, et al. Peri-operative complications following surgical closure of atrial septal defect type II in 232 patients - a baseline study. Eur Heart J 1994; 15: 1381-4.

18. Brochu MC, Baril JF, Dore A, et al. Improvement in exercise capacity in asymptomatic and mildly symptomatic adults after atrial septal defect percutaneous closure. Circulation 2002; 106: 1821-6.

19. Roos-Hesselink JW, Meijboom FJ, Spitaels SE, et al. Excellent survival and low incidence of arrhythmias, stroke and heart failure long-term after surgical ASD closure at young age. A prospective follow-up study of 21-33 years. Eur Heart J 2003; 24: 190-7.

20. Konstantinides S, Geibel A, Olschewski M, et al. A comparison of surgical and medical therapy for atrial septal defect in adults. N Engl J Med 1995; 333: 469-73.

21. Horvath KA, Burke RP, Collins JJ, Cohn LH. Surgical treatment of adult atrial septal defect: early and long-term results. J Am Coll Cardiol 1992; 20: 1156-9.

22. Yüce $M$, Ozer O, Cakici M, et al. Closure of secundum atrial septal defects by the Amplatzer occluder device. Turk Kardiyol Dern Ars 2011; 39: 35-40. 